\title{
Avenues of cognition of nongravitational local gauge field theories
}

\author{
Peter Minkowski ${ }^{1, a}$ \\ ${ }^{1}$ Albert Einstein Center for Fundamental Physics - ITP, University of Bern, Switzerland
}

\begin{abstract}
This controbution is devoted to present basic fearures of a unifying local gauge field theory, prevailing up to a mass scale of approximately $10^{16} \mathrm{GeV}$, allowing the neglect of gravitational curvature effects - indicated by the attribute : 'nongravitational' in the title above .
\end{abstract}

\section{Introduction}

The two main mass generation mechanisms within a general gauge field theory in $3+1$ uncurved space-time dimensions - henceforth called gravitationless gauge field theory -

- minimally the neutrino mass extended standard model based on the gauge group $S U 3_{c} \times S U 2_{L} \times U 1_{y}$ and one scalar doublet with respect to $S U 2_{L}-$

form the basis of the present outline, supposed to be an integral part of an enveloping unifying gauge group, minimally $\mathrm{SO} 10 \equiv \operatorname{spin} 10$.

Table 1.

1) the Bose condensation of some components of elementary scalar fields scalar stands here for scalar and/or pseudoscalar Yukawa couplings

2) the Bose condensation of the gauge field-strength bilinear

- gauge- and renormilzation group invariant with respect to the unbroken gauge group $S U 3_{c}$

The key features of point 1) in table 1, are outlined in section 2 .

The main topic here, point 2) in table 1, is elaborated on in section E.

\footnotetext{
ae-mail: mink@itp.unibe.ch
} 


\section{Bose condensation of elementary scalar fields - the Brout-Englert-Higgs effect}

Recent assessments can be found in the talks of François Englert - [1-2013] and, more historically oriented, Peter Higgs [2-2013] at the Nobel Prize 2013 awards ceremony.

We base the general properties of spontaneous gauge breaking of an enveloping gravitationless gauge field theory, based on a gauge group $G_{e n v} \supset G_{\min }$ in the sense of gauge group unification beyond the minimal case $G_{\min }=S U 3_{c} \times S U 2_{L} \times U 1_{y}$, presented in the introduction.

A minimal such enveloping gauge group is

$$
G_{e n v}^{\min }=\mathrm{SO} 10 \equiv \operatorname{spin} 10
$$

as discussed in refs. [3-1975] and [4-2008] .

Within larger simple enveloping groups the exceptional chain

$$
G_{e n v} \rightarrow E 6 \subset E 8 \supset E 6 \times S U 3
$$

is singled out [5-1976], [6-1980], most importantly because it offers the possibility of canceling all gauge- and gravitational anomalies in the product gauge group [7-1984] , [8-2012]

$$
G_{e n v}=E 8 \times E 8
$$

Following the hypothesis of an underlying unifying gauge group the top down of gauge breaking is initiated by a primary breaking followed last by the electroweak gauge breaking .

\section{2-1 Primary gauge breaking}

Primary gauge beaking is linked to the unifying gauge group scale $M_{\text {env }}$ assumed and also restricted by limits on direct observation of baryon decays and lepton flavor violation to be much larger than the electroweak scale

$$
M_{\text {env }} \gg v=\left(\sqrt{\sqrt{2} G_{F}}\right)^{-1}=246.220 \mathrm{GeV}
$$

In eq. $4 \mathrm{v}$ denotes the v.e.v. of the unique doublet scalar field using the quaternion associated basis for the local scalar fields

$$
\begin{aligned}
& z(x)=\left(\begin{array}{cc}
\varphi^{0} & -\left(\varphi^{-}\right)^{*} \\
\varphi^{-} & \left(\varphi^{0}\right)^{*}
\end{array}\right)(x) \\
& \varphi^{0}=1 / \sqrt{2}\left(Z_{0}-i Z_{3}\right) ; \varphi^{-}=1 / \sqrt{2}\left(Z_{2}-i Z_{1}\right) \\
& Z_{\mu}=\left(Z_{\mu}\right)^{*}=\left(Z_{0}, Z_{1}, Z_{2}, Z_{3}\right)=\left(Z_{0}, \vec{Z}\right) \\
& \sigma_{0}=\left(\begin{array}{ll}
1 & 0 \\
0 & 1
\end{array}\right), \sigma_{1}=\left(\begin{array}{ll}
0 & 1 \\
1 & 0
\end{array}\right), \sigma_{2}=\left(\begin{array}{rr}
0 & -i \\
i & 0
\end{array}\right), \sigma_{3}=\left(\begin{array}{rr}
1 & 0 \\
0 & -1
\end{array}\right) \\
& \sigma_{\mu}=\left(\sigma_{0}, \sigma_{1}, \sigma_{2}, \sigma_{3}\right)=\left(\sigma_{0}, \vec{\sigma}\right)
\end{aligned}
$$

In eq. 5 the symbol * denotes hermitian conjugation of individual complex and/or real field components.

Thus the quantity $\mathrm{z}$, defined in eq. 5 , shows its quaternionic representation

$$
z(x)=\frac{1}{\sqrt{2}}\left(Z_{0} \sigma_{0}+\sum_{k=1}^{3} Z_{k} \frac{1}{i} \sigma_{k}\right)(x)
$$


The four $2 \times 2$ matrices, displayed in eq. 5 , form a 1 to 1 true representation of the base quaternions

$$
\begin{aligned}
& \sigma_{0} \leftrightarrow q_{0}=\mathbb{I I} ; \frac{1}{i} \sigma_{m} \leftrightarrow q_{m} \text { for } m=1,2,3 \\
& q_{m} q_{n}=-\delta_{m n} q_{0}+\varepsilon_{m n r} q_{r} \text { for } m, n, r=1,2,3
\end{aligned}
$$

As we will see, the final stage of the ( nu-mass extended - ) standard model gauge breaking involving just 1 doublet of scalars with respect to the elecroweak part $S U 2_{w} \times \mathcal{Y}_{w}$ represents a case for perfectly semiclassical, driven Bose condensation, eventually contrasting with intrinsic properties of primary breakdown.

This is so, because there is precisely 1 invariant

$$
I\left(z, z^{\dagger}\right)=z z^{\dagger}=\frac{1}{2} \sum_{\mu=0}^{3}\left(Z_{\mu}\right)^{2}
$$

with respect to $S U 2_{w} \times \mathcal{y}_{w}$ of which a general invariant is a function .

This would not remain true for more than one scalar doublet .

As a consequence of eqs. $4-8$, the electroweak gauge breaking is driven and semiclassical

$$
\langle\Omega|z(x)| \Omega\rangle=\frac{1}{\sqrt{2}}\left(\begin{array}{ll}
v & 0 \\
0 & v
\end{array}\right) \text { with } v=246.220 \mathrm{GeV} \text {, independent of } x
$$

We shall discuss 2 types of primary gauge breaking denoted a) and b) below .

The v.e.v. $v$ in eq. 9 corresponds to the classical minimum of the quartic potential, uniquely restricted to depend on two parameters

$$
V\left(z, z^{\dagger}\right)=\left[-\mu^{2} z z^{\dagger}+\lambda\left(z z^{\dagger}\right)^{2}\right]_{11}
$$

The minimum conditions become

$$
\begin{aligned}
\partial_{Z_{v}} V & =\left(-\mu^{2}+\lambda|Z|^{2}\right) Z_{v}=\left.0 \rightarrow z z^{\dagger}\right|_{11}=\frac{1}{2} \mu^{2} / \lambda \\
\left.V\right|_{\text {min }} & =-\frac{1}{4}\left(\mu^{4} / \lambda\right)
\end{aligned}
$$

The second derivatives with respect to $\mathrm{Z}$ at the minimum of the potential become

$$
\frac{1}{2} \partial_{Z_{\varrho}} \partial_{Z_{\sigma}} V=\left.\lambda Z_{\varrho} Z_{\sigma}\right|_{\min }=\lambda v^{2} \delta_{0 \varrho} \delta_{0 \sigma} ;\left.Z_{v}\right|_{\min }=(v, \overrightarrow{0})
$$

Expanding the deviation of the potential up to quadratic terms around the minimum thus yields

$$
(\Delta V)^{(2)}=\lambda v^{2}\left(\Delta Z_{0}\right)^{2} ; Z_{0}(x)=v+\Delta Z_{0}(x)
$$

It is customary to denote the shifted hermitian field $\Delta Z_{0}(x)$

$$
Z_{0}=v+\Delta Z_{0} ; \Delta Z_{0}(x)=H(x)
$$

From eq. 14 we read off the mass of the field $H(x)$ as well as the vanishing of the mass of the other three fields

$$
m_{H}^{2}=2 \lambda v^{2}=2 \mu^{2} ;\left.m_{\vec{Z}}\right|_{\text {from } V}=0 ; \vec{Z}(x)=\left(Z_{1}, Z_{2}, Z_{3}\right)(x)
$$

However in the case at hand the existence of would-be long range forces represented by the $S U 2{ }_{L} \times$ $U 1$ y gauge field interactions does not permit the existence of goldstone-modes .

The three would-be Goldstone fields, defined in eq. 15, through their space-time gradient, mix with the gauge bosons to become massive, vis. $W^{ \pm}$and $\widetilde{Z}$

$$
\partial_{x \tau} Z_{m}(x) \leftrightarrow W_{\tau}^{ \pm}(x), \widetilde{Z}_{\tau}(x)
$$


in such a way as to obtain masses of the 3 massive gauge bosons $m_{W}, m_{\widetilde{Z}}$ and physically form the longitudinal spin components of the resulting massive states (resonances). In eq. 16 the neutral massive gauge boson is denoted $\widetilde{Z}$ not to confuse it with the sclar field components $Z_{0}, \vec{Z}$.

In tree approximation the mass-square of the $\mathrm{H}$-field is twice the value of the $Z_{0}$ field in the unbroken case, i.e. for $\mu^{2} \rightarrow-\mu^{2}$

$$
m_{H}^{2}=2 \mu^{2} \rightarrow \mu=\frac{1}{\sqrt{2}} m_{H}=88.388 \mathrm{GeV} \text { for } m_{H}=125 \mathrm{GeV}
$$

The detailed description of the mixing as stated in eq. 16 is not given here. A complete derivation can be found in the textbook ref. $9=[9-1995]$.

The simplicity of the presumably lowest in scale gauge breaking relative to the SM gauge group $S U 2_{L} \times U 1$ y prompted most discussions of primary gauge breaking to be of the same type b) presented below, i.e. driven - semiclassical: for an example of primary gauge breakdown patterns see e.g. ref. $10=[10-1013]$.

This brings us to the two types a) and b) - of an enveloping gravitationless gauge field theory. For both types the scalar field variables turn out to involve a complex ensemble of irreducible representations of the enveloping gauge group - as e.g. SO10, in particular for the generation of masses for neutrino flavors, both light and heavy, as discussed e.g. in refs. $4=[4-2008]$ and $10=[10-1013]$ through Yukawa couplings to basic fermion bilinears .

Two recent papers deserve mention here by Guido Altarelli ref.11 $=[11-2014]$ and Ferruccio Feruglio, Ketan M. Patel and Denise Vicino ref. 12 = [12-2014]

We introduce notation for the ensemble of scalar fields adapted to primary gauge breaking, generalizing $S U 2{ }_{L}$ doublets as defined in eqs. 5 - 7 .

Lets fix for definiteness

$$
G_{e n v}^{\min }=\mathrm{SO} 10 \equiv \operatorname{spin} 10
$$

in the following. A general irreducible representation of SO10 shall be denoted $[\mathcal{D}]$, where $\mathcal{D}$ is equivalenced to its dimension. As entry point we take the [16] representation for one fermion family ( the lightest in mass ) in the left chiral basis

$$
\text { [16] : }\left(\begin{array}{ccccccccc}
u^{1} & u^{2} & u^{3} & v_{e} & \mathcal{N}_{e} & \widehat{u}^{3} & \widehat{u}^{2} & \widehat{u}^{1} \\
d^{1} & d^{2} & d^{3} & e^{-} \mid & e^{+} & \widehat{d}^{3} & \widehat{d}^{2} & \widehat{d}^{1}
\end{array}\right)^{\dot{\gamma} \rightarrow L}
$$

It is instructive to illustrate the use of the basis defined in eq. 19 considering

\section{the Majorana logic characterized by $\mathcal{N}_{e, \mu, \tau}$}

derived from the subgroup decomposition

$$
\operatorname{spin} 10 \rightarrow \text { SU5 } \times \mathrm{U}_{J_{5}}
$$

Among the 3 generators of spin10 commuting with $S U 3_{c}, I_{3 L}, I_{3 R}, B-L$ and forming part of the Cartan subalgebra of spin10 there is one combination, denoted $J_{5}$ in eq. 20, commuting with its largest unitary subgroup SU5. The charges $Q\left(J_{5}\right)$ form the pattern as in eq. 21

$$
\text { [16] : } Q\left(J_{5}\right)=\left(\begin{array}{rrrrrrrrr}
1 & 1 & 1 & -3 & \mid & 5 & 1 & 1 & 1 \\
1 & 1 & 1 & -3 & \mid & 1 & -3 & -3 & -3
\end{array}\right)
$$

$Q\left(J_{\mu}\right)$ with charges as given in eq. 21 represents a hermitian generator of the Cartan subalgebra of spin10, unique up to a (real) multiplicative factor, which commutes with the the SU5 subgroup of 
spin10. The flavors within one family sharing the same Q-charges in fact form irreducible representations of SU5, which shall be labeled

$$
\mathcal{D}(S U 5) \rightarrow\{\mathcal{D}(S U 5)\}_{Q}
$$

with $\mathcal{D}$ ( $S U 5$ ) equivalenced with the dimension of the representation. The suffix $\mathrm{Q}$ is added in eq. 22 , since in the present context SU5 multiplets necessarily occur embedded in SO10 representations. Thus the $\mathrm{Q}$ values of the [16] representation on the right han side of eq. 21 translate to

$$
[16]=\{1\}_{5}+\{10\}_{1}+\{\overline{5}\}_{-3}
$$

The sequence of $\mathrm{Q}$ values : $Q=5,1,-3$ as arranged in the sequence on the right hand side of eq. 23 - decreasing in steps of 4 - is related to the properties of binomial coefficients

$$
\left(\begin{array}{c}
10 \\
n
\end{array}\right) \text { for } n=0,4,8 \text { and } n=10,6,2
$$

with $\mathcal{D}$ ( SU5 ) equivalenced with the dimension of the representation. The suffix $\mathrm{Q}$ is added in eq. 22 , since in the present context SU5 multiplets necessarily occur embedded in SO10 representations. This is derived in ref. $4-$ [4-2008] .

It is instructive to decompose the Q-value pattern in eq. 21 into an $S U 2_{L+R}$ invariant part and the remainder proportional to $I_{3} L+R$

$$
\begin{aligned}
& \left(\begin{array}{rrrrrrrrr}
1 & 1 & 1 & -3 & \mid & 5 & 1 & 1 & 1 \\
1 & 1 & 1 & -3 & \mid & 1 & -3 & -3 & -3
\end{array}\right)= \\
& \left(\begin{array}{rrrrrrrrr}
1 & 1 & 1 & -3 & \mid & 3 & -1 & -1 & -1 \\
1 & 1 & 1 & -3 & \mid & 3 & -1 & -1 & -1
\end{array}\right)+ \\
& \left(\begin{array}{rrrrrrrrr}
0 & 0 & 0 & 0 & \mid & 2 & 2 & 2 & 2 \\
0 & 0 & 0 & 0 & \mid & -2 & -2 & -2 & -2
\end{array}\right)
\end{aligned}
$$

From eq. 25 we obtain the identification of the spin10 Cartan subalgebra hermitian components ( charges )

$$
Q=3(B-L)-4 I_{3 R}
$$

In eq. $26 \mathrm{~B}$ and $\mathrm{L}$ denote baryon and lepton number respectively .

Two remarks shall follow, concerning the recognizable key features inherent to spontaneous gauge breaking of an enveloping gravitationless gauge field theory, based on a gauge group $G_{e n v}=\operatorname{spin} 10$

1) primary gauge breakdown

must be much different than on the lowest - i.e. electroweak - scale level pertaining to $G_{S M}=$ $S U 3_{c} \times S U 2_{L} \times U 1_{y}$.

This is so because empirically well established candidate symmetries, like baryon and lepton number conservation are broken on the primary level and imply very large scale of unification $M_{\text {env }}=$ $O\left(10^{16} \mathrm{GeV}\right)$.

As examples let me quote the upper limits of the $\mu^{+} \rightarrow e^{+} \gamma$ and $\mu^{+} \rightarrow e^{+}+e^{+} e^{-}$branching fractions

$$
\begin{aligned}
& \operatorname{Br}\left(\mu^{+} \rightarrow e^{+}+\gamma\right)<2.4 .10^{-12} \\
& \operatorname{Br}\left(\mu^{+} \rightarrow e^{+}+e^{+} e^{-}\right)<1.0 .10^{-12} \text { in ref. } 11-[13-2013]
\end{aligned}
$$


2) the power of the set of scalar fields

involved in primary gauge breaking does not follow any principle of minimal selection of spin10 representations pertaining to scalar fields .

\section{specific notation for scalar field variables}

We proceed defining notation for scalar field variables suitable for primary gauge breaking

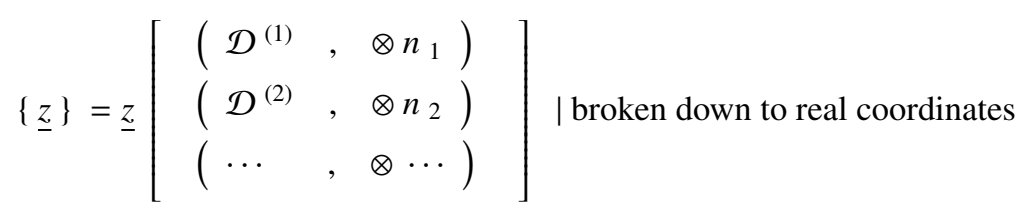

In eq. $28 \mathcal{D}^{(v)} ; v=1,2, \cdots$ denote a complete set of unitary irreducible representations of spin 10 , finite dimensional; constructively defined through the method of Peter and Weyl [14-1927] .

$n_{v}$ stands for the multiplicity of a given representation $\mathcal{D}^{(v)}$.

For $\mathcal{D}^{(v)}, \overline{\mathcal{D}}^{(v)}$ beeing a pair of inequivalent, relative complex conjugate representations the coordinates

$$
\underline{z}\left(\mathcal{D}^{(v)}\right), \bar{z}\left(\overline{\mathcal{D}}^{(v)}\right)
$$

broken down to real and imaginary parts count as 2 (complex) dim ( $\mathcal{D}$ ) components over real numbers. This is the meaning of the attribute 'broken down to real coordinates' on the right hand side of eq. 28 .

Thus choosing real values for the components of $\underline{z} \in R^{M}$ as defined in eq. 28 it follows

$$
\underline{z}=\left(z_{1}, \cdots, z_{r}, \cdots, z_{M}\right) ; z_{r}: \text { hermitian fields }
$$

we find

$$
M=\sum_{v} n_{v}\left\{\begin{array}{l}
\operatorname{dim}\left(\mathcal{D}^{(v)}\right) \text { for } \mathcal{D}^{(v)} \text { real } \\
2 \text { complex } \operatorname{dim}\left(\mathcal{D}^{(v)}\right) \text { for } \mathcal{D}^{(v)} \text { complex }
\end{array}\right\}<\infty
$$

\section{to get an idea of the power of the set of scalars}

We illustrate the order of M , in eqs. 29, 30 by the representations of the fermion bilinears from left and right chiral bases, adapting the scalar variables to their definition in eq. 29 , allowing for complex linear combinations applied to complex representations ( from section E and ref. 4 - [4-2008] ).

$$
\begin{aligned}
& \mathcal{H}_{\text {fermion mass }} \longleftarrow \\
& \left(\bar{z}^{\overline{126} F G}\right)_{\bar{\xi}}\left(f_{a 16}\right)_{\dot{\gamma}}\left(f_{b} 16 G\right)^{\dot{\gamma}} C\left(\begin{array}{c|cc}
126 & 16 & 16 \\
\xi & a & b
\end{array}\right)+\text { h.c. } \\
& \left(\bar{z}^{\overline{126} F G}\right)_{\bar{\xi}}: \text { (pseudo-) scalar fields in the } \overline{126} \text { representation of SO10 } \\
& F, G=I, I I, I I I \text { : fermion family indices }
\end{aligned}
$$


In eq. $31 C\left(\begin{array}{c|cc}126 & 16 & 16 \\ \xi & a & b\end{array}\right)$

denotes the spin10 Clebsch-Gordan coefficients projecting the product of two ( fermionic ) 16 representations on irreducible spin10 representations .

We reproduce the 4 product representations of 16 and $\overline{16}$ representations from ref. 4 (Appendix E) $=[4-2008]$ )

\begin{tabular}{|c|c|c|}
\hline & [16] & $\overline{16}$ \\
\hline [16] & $s: \begin{array}{l}{[10]+} \\
{[126]}\end{array}, a:[120$ & $\begin{array}{l}{[1]+[45]+} \\
{[210]}\end{array}$ \\
\hline$[\overline{16}]$ & $\begin{array}{l}{[1]+[45]+} \\
{[210]}\end{array}$ & $\left.s:{ }^{[10]+} \overline{126}\right], a:[120]$ \\
\hline
\end{tabular}

The real and complex representations in the multiplication table in eq. 32 are denoted $\mathcal{D}_{R}, \mathcal{D}_{C}$ respectively

$$
\begin{aligned}
& \mathcal{D}_{R}:[10]_{R},[120]_{R},[1]_{R},[45]_{R},[210]_{R} \\
& \mathcal{D}_{C}:[126]_{C},[\overline{126}]_{C}
\end{aligned}
$$

Choosing minimally multiplicities 2 for real and 1 for complex representations in eqs. 32 and 33 yields

$$
M=772+252=1028
$$

We conclude from the example multiplicities leading to eq. 34

$$
M \geq O(1000)
$$

\section{2-1-a Primary gauge breaking - type a)}

The gauge breaking in type a) gravitationless gauge field theory is

1) driven

by pre-established quadratic, cubic and quartic scaler field self interactions

2) not reducible to semiclassical approximation

for vacuum expected values for scalar variables and their composite operators, necessarily include gauge variant ones in order to qualify for gauge breaking

For clarity let me remark that condition 2) above is necessary, since in the case of exclusively gauge invariant vev's for composite scalar operators they are part of an alternative case - in conjunction with other gauge invariant composite field variables - of spontaneous mass generation without gauge breaking. This will be discussed within QCD with 3 light flavors - without scalars - elsewhere .

\section{2-1-b - Primary gauge breaking - type b)}

The gauge breaking in type b) gravitationless gauge field theory is

1) driven

by pre-established quadratic, cubic and quartic scaler field self interactions like in type a)

2) reducible to semiclassical approximation

for vacuum expected values for scalar variables and their composite operators. This is the usual case discussed in the literature, as e.g. in refs. $10=[10-1013]-$ and $15=[15-2013]$, while in the latter reference the main topic is electroweak gauge breaking . 
The semiclassical approximation - with respect to vev's of scalar fields and their composite local operators as well as composite operators involving other fields - means

$$
\langle\Omega|f(\underline{z})| \Omega\rangle=f(\langle\Omega|\underline{z}| \Omega\rangle)
$$

\section{E The spin10 product representations $(16 \oplus \overline{16}) \otimes(16 \oplus \overline{16})$}

We follow the spin10 decomposition discussed in section 2-1 (eq. 37 repeated below )

$$
\operatorname{spin} 10 \rightarrow \text { SU5 } \times \mathrm{U}{ }_{5}
$$

Further let us denote representations of spin10 as opposed to those pertaining to SU5 and associated $J_{5}$ quantum number by

$$
\operatorname{spin} 10:[\operatorname{dim}] ; \mathrm{SU} 5 \times \mathrm{U}_{J_{5}}:\{\operatorname{dim}\}_{J_{5}}
$$

Thus we obtain

$$
\begin{aligned}
& {[16]=\{1\}_{+5}+\{10\}_{+1}+\{\overline{5}\}_{-3}} \\
& {[\overline{16}]=\{1\}_{-5}+\{\overline{10}\}_{-1}+\{5\}_{+3}}
\end{aligned}
$$

In turn SU5 representations shall be decomposed along the standard model gauge group SU3 $c \otimes$ $\mathrm{SU} 2{ }_{L} \otimes \mathrm{U} 1 y$, where $y$ denotes the electroweak hypercharge (with a factor $\frac{1}{2}$ included)

$$
\begin{gathered}
y=Q_{e . m .} / e-I_{3 L} \\
\left.\{\operatorname{dim}\} \rightarrow \sum\right]\left(\operatorname{dimSU}_{c}, \operatorname{dimSU} 2_{L}\right)_{y}[
\end{gathered}
$$

The brackets on the right hand side of eq. 41 are reversed in order not to confuse spin 10 - and standard model representations.

Then the base $16(\overline{16})$ decompose to

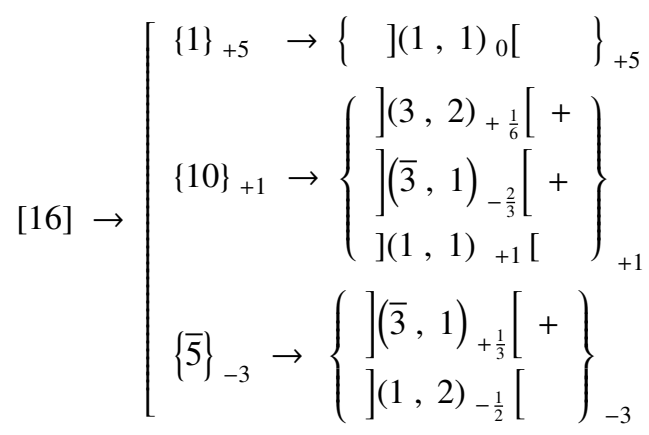

The product representations $(16 \oplus \overline{16}) \otimes(16 \oplus \overline{16})$ generate all SO10 antysymmetric tensor ones, of which we encountered the fivefold antisymmetric in section 2-1.

To elaborate we specify the $\mathrm{n}$-fold antisymmetric tensors obtained from the 10-representation of SO10

$$
\begin{aligned}
& {\left[t_{0}\right] \sim 1} \\
& {\left[t_{1}\right]^{A} \sim z^{A} ; A=1,2, \cdots, 10 \leftrightarrow\left[t_{1}\right]=\{\overline{5}\}_{2} \oplus\{5\}_{-2}} \\
& {\left[t_{2}\right]^{\left[A_{1} A_{2}\right]} \sim \frac{1}{2}\left(z_{1}^{A_{1}} z_{2}^{A_{2}}-z_{1}^{A_{2}} z_{2}^{A_{1}}\right)} \\
& \quad \cdots \\
& {\left[t_{n}\right]^{\left[A_{1} A_{2} \cdots A_{n}\right]} \sim \frac{1}{n !} \sum \operatorname{sgn}\left(\begin{array}{ccc}
1 & \cdots & n \\
\pi_{1} & \cdots & \pi_{n}
\end{array}\right) z_{1}^{A_{\pi_{1}}} z_{2}^{A_{\pi_{2}}} \cdots z_{n}^{A_{\pi_{n}}}} \\
& n \leq 10
\end{aligned}
$$


The quantities $\left[t_{n}\right]$ defined in eq. 43 form irreducible real representations of SO10, except for $\mathrm{n}=$ 5 , which is composed of the relatively complex irreducible representations 126 and $\overline{126}$.

The tenfold antisymmetric invariant corresponds to $\left[t_{n=10}\right]$. The product of two full Clifford algebras pertaining to spin10 contains all $\left[t_{n}\right] ; n=0 \cdots 10$ representations exactly once .

Treating the $\mathrm{n}=5$ tensor as one representation - it is reducible only over $\mathbb{C}$ - the dimensions of the $\left[t_{n}\right]$ representations follow Pascal's triangle .

$\begin{array}{ccccccccccc}{\left[t_{0}\right]} & & {\left[t_{2}\right]} & & {\left[t_{4}\right]} & & {\left[t_{6}\right]} & & {\left[t_{8}\right]} & & {\left[t_{10}\right]} \\ & {\left[t_{1}\right]} & & {\left[t_{3}\right]} & & {\left[t_{5}\right]} & & {\left[t_{7}\right]} & & {\left[t_{9}\right]} & \\ 1 & & 45 & & 210 & & 210 & & 45 & & 1 \\ & & & 120 & & 252 & & 120 & & 10 & \end{array}$

This corresponds to the following products of $16+\overline{16}$

\begin{tabular}{|c|c|c|c|}
\hline & & [16] & {$[\overline{16}]$} \\
\hline [16] & $s:$ & $\begin{array}{l}{[10]+} \\
{[126]}\end{array}, a:[120]$ & $\begin{array}{l}{[1]+[45]+} \\
{[210]}\end{array}$ \\
\hline$[\overline{16}]$ & & $\begin{array}{l}{[1]+[45]+} \\
{[210]}\end{array}$ & $s: \begin{array}{l}{[10]+} \\
{[\overline{126}], a:[120]}\end{array}$ \\
\hline
\end{tabular}

The correspondence of product representations of the $16+\overline{16}=32$ associative Clifford algebra with the sum of antisymmetric tensor ones follows from the completeness of all products of $\gamma$ matrices forming the spin 10 algebra i.e. are of dimension

$$
(32)^{2}=\left(2^{5}\right)^{2}=2^{10}
$$

We proceed to reduce the [16] $\otimes[16]$ product with respect to $J_{5}, \mathrm{SU} 5$ and $\mathrm{SU} 3_{c} \times \mathrm{SU} 2_{L} \times \mathrm{U} 1 y$. The individual products are $(s(a):$ (a)symmetric $)$

\begin{tabular}{c|ccc} 
& $\{1\}_{5}$ & $\{10\}_{1}$ & $\{\overline{5}\}_{-3}$ \\
\hline$\{1\}_{5}$ & $\{1\}_{10 s}$ & $\{10\}_{6}$ & $\{\overline{5}\}_{2}$ \\
$\{10\}_{1}$ & $\{10\}_{6}$ & $\left(\begin{array}{l}\{\overline{5}\}_{2}+ \\
\{\overline{50}\}_{2}\end{array}\right)_{s}\left(\{\overline{45}\}_{2}\right)_{a}$ & $\left(\begin{array}{l}\{5\}_{-2}+ \\
\{45\}_{-2}\end{array}\right)$ \\
$\{\overline{5}\}_{-3}$ & $\{\overline{5}\}_{2}$ & $\left(\begin{array}{l}\{5\}_{-2}+ \\
\{45\}_{-2}\end{array}\right)$ & $\left(\{\overline{15}\}_{-6}\right)_{s}\left(\{\overline{10}\}_{-6}\right)_{a}$
\end{tabular}


Decomposing the diagonal $\{\mathrm{SU} 5\}_{J_{5}}$ representations ( eq. 42 ) yields

$$
\begin{aligned}
& \left(\{10\}_{1} \otimes\{10\}_{1}\right)_{s}=\{\overline{5}\}_{2}+\{\overline{50}\}_{2} \quad \downarrow
\end{aligned}
$$

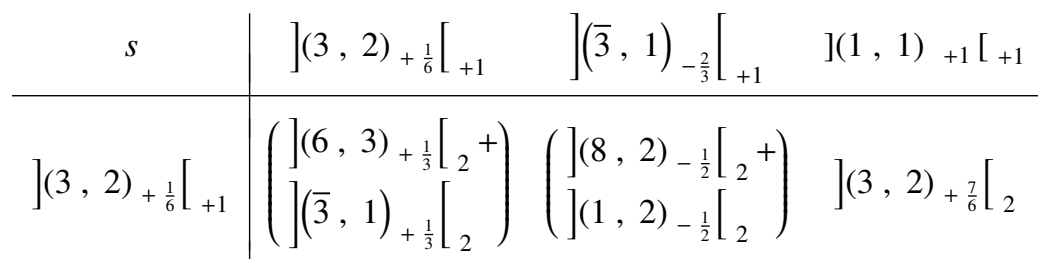

$$
\begin{aligned}
& \begin{array}{lll}
](\overline{3}, 1)_{-\frac{2}{3}}\left[{ }_{+1}\right. & ](\overline{6}, 1)_{-\frac{4}{3}}[2 & ](\overline{3}, 1)_{+\frac{1}{3}}\left[{ }_{2}\right. \\
](1,1)_{+1}\left[{ }_{+1}\right. & ](1,1)_{+2}[2
\end{array} \\
& \left(\{\overline{5}\}_{-3} \otimes\{\overline{5}\}_{-3}\right)_{s}=\{\overline{15}\}_{-6} \quad \downarrow
\end{aligned}
$$

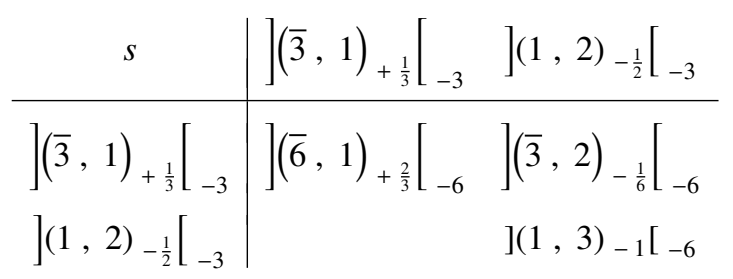

$$
\begin{aligned}
& \downarrow \\
& \text { complex e.w. triplet coupling to } \\
& \frac{1}{2}\left(v_{\dot{F}}^{*}\right)^{\alpha}\left(v_{\dot{G}}^{*}\right)_{\alpha}
\end{aligned}
$$

Next we assemble the (anti)symmetric products $([16] \otimes[16])_{s}=[10] \oplus[126]$ and $([16] \otimes[16])_{a}=[120]$ with respect to SU5 $\otimes \mathrm{U}{ }_{J_{5}}$ using eq. 47

$$
\begin{gathered}
([16] \otimes[16])_{s}=[10] \oplus[126] \quad \downarrow \\
\left.\qquad \begin{array}{c}
\{5\}_{-2}+ \\
\left\{\overline{5}_{I}\right\}_{2}
\end{array}\right] \\
\oplus\left[\begin{array}{c}
\left.\{1\}_{10}+\left\{\overline{5}_{I I}\right\}_{2}+\{10\}_{6}+\{\overline{15}\}_{-6}\right] \\
+\{45\}_{-2}+\{\overline{50}\}_{2}
\end{array}\right] \\
([16] \otimes[16])_{a}=[120] \\
=\left[\begin{array}{c}
\{5\}_{-2}+\{\overline{5}\}_{2} \\
+\{10\}_{6}+\{\overline{10}\}_{-6} \\
+\{45\}_{-2}+\{\overline{45}\}_{2}
\end{array}\right]
\end{gathered}
$$

The roman indices $\quad I, I I$ in eq. 50 indicate that appropriate linear combinations of the two $\{\overline{5}\}_{2}$ representations form parts of [10] and [126] respectively . 
It remains to decompose the SU5 $\otimes \mathrm{U} 1_{J_{5}}$ representations in eq. 50 with respect to $\mathrm{SU} 3_{c} \times \mathrm{SU} 2_{L} \times$ $\mathrm{U} 1 y$. We do this associating according to the product representations as they appear in eq. 50

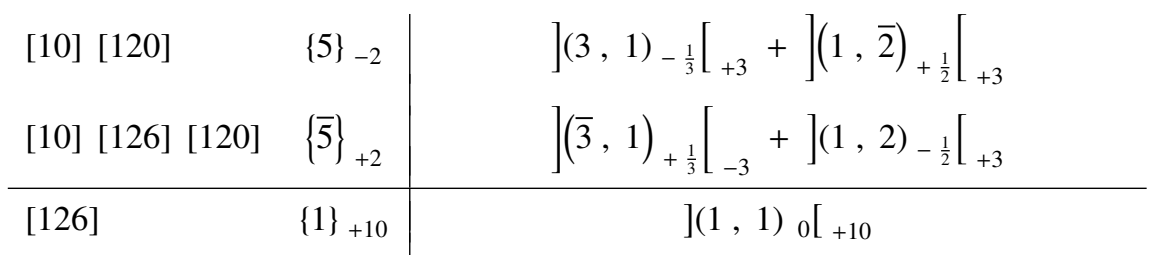

$$
\begin{aligned}
& \left.\left.[126][120] \quad\{10\}_{+6}\right]\right](3,2)_{-\frac{1}{6}}\left[{ }_{+6}+\right](\overline{3}, 1)_{-\frac{2}{3}}\left[_{+6}+\right](1,1)_{+1}[+6 \\
& \left.[120] \quad\{\overline{10}\}_{-6} \mid\right](\overline{3}, \overline{2})_{+\frac{1}{6}}\left[_{-6}+\right](3,1)_{+\frac{2}{3}}\left[_{-6}+\right](1,1)_{-1}[-6 \\
& \left.[126] \quad\{\overline{15}\}_{-6} \mid\right](\overline{6}, 1)_{+\frac{2}{3}}\left[_{-6}+\right](\overline{3}, 2)_{-\frac{1}{6}}\left[_{-6}+\right](1,3)_{-1}\left[{ }_{-6}\right. \\
& {[126][120] \quad\{45\}_{-2}}
\end{aligned}
$$

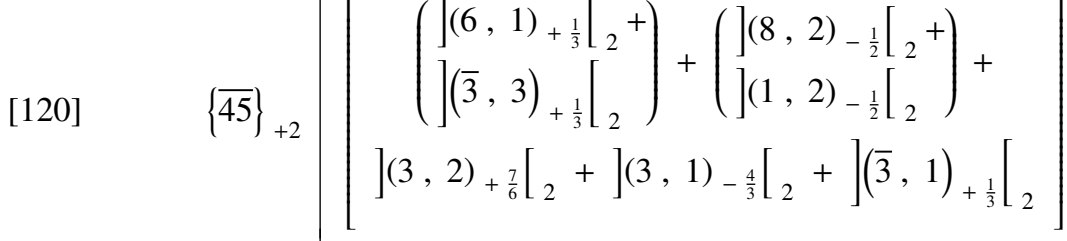

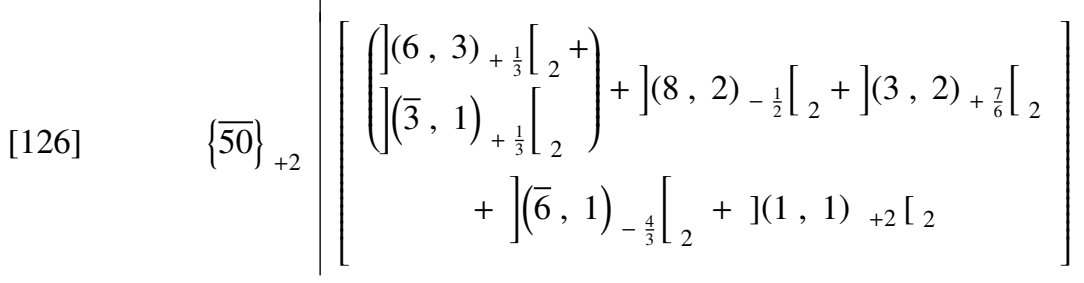

$$
\begin{aligned}
& \left(\{10\}_{1} \otimes\{10\}_{1}\right)_{s}=\{\overline{45}\}_{2} \quad \downarrow
\end{aligned}
$$

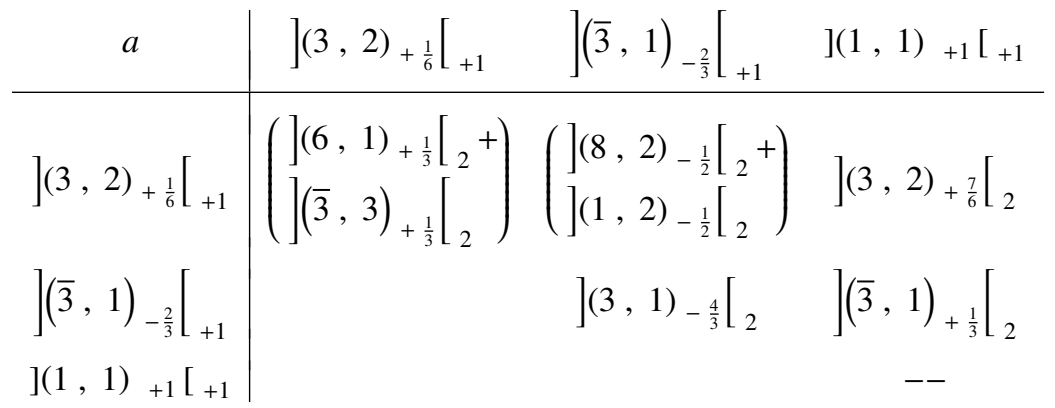


I end the collection of representation decompositions with the adjoint [45] representation of SO10 $([10] \otimes[10])_{a}=[45]$

$\left.\begin{array}{c|cc}a & \{5\}_{-2} & \{\overline{5}\}_{+2} \\ \hline\{5\}_{-2} & \{10\}_{-4} & \{1\}_{0} \leftrightarrow J_{5} \\ \{24\}_{0} \leftrightarrow \text { adjoint SU5 }\end{array}\right\}$

It should be noted that despite coinciding dimensions the following entities are most distinct

$$
[10] \neq\{10\}_{-4}, \quad\{10\}_{6} \quad ; \quad[45] \neq\{45\}_{-2} ; \cdots
$$

\section{Synthesis of 'Beyond the Standard Model Physics' from the basic of a unifying gauge theory, in particular spin10}

I wish to emphasize in conclusion of the previous derivational sections a few central points here:

1) The timeline of 'Unified theories of leptons and hadrons', ref. $3=$ [3-1975]

Together with Harald Fritzsch we initiated, towards the end of 1973, an in-depth imvestigation into characteristic features of unified interactions of leptons and quarks, based on an associated gravitionless simple gauge group.

In November 2014 the closed charm particle $J / \psi$ was discovered, ref. $16=[16-1974]$ at BNL with the reaction $p \mathrm{Be} \rightarrow e^{+} e^{-} x$ and ref. $17=[17-1974]$ at SLAC in the reaction $e^{+} e^{-} \rightarrow e^{+} e^{-} x$.

In the aftermath of enthusiasm and excitement, accompanied by a plethora of papers trying and for their majority succeeding to interpret this discovery, we decided to finish the mentioned investigation without adopting any major topical revisions.

It may be due to these circumstances that the messages contained in ref. $3=[3-1975]$ were only understood in part.

2) gauge bosons completing SO10 beyond $S U 3_{c} \times S U 2_{L} \times U 1_{y}$

The adjoint representation of SO10 can be broken down along the decomposition chain

$$
\mathrm{SO} 10 \rightarrow \text { SU5 } \times U 1_{J}
$$

to yield the quantum numbers

$$
\begin{aligned}
& \operatorname{Adj}(\mathrm{SO} 10)=\left(\{\overline{5}\}_{+2} \oplus\{5\}_{-2}\right) \otimes^{a}\left(\{\overline{5}\}_{+2} \oplus\{5\}_{-2}\right) \\
& \left.\{\overline{5}\}_{2}=\right](\overline{3}, 1)_{+\frac{1}{3}}\left[{ }_{2} \oplus\right](1,2)_{+\frac{1}{2}}[2
\end{aligned}
$$

For the product in eq. 57 it thus follows

$$
\operatorname{Adj}(\operatorname{SO} 10)=\left[\begin{array}{l}
](3,1)_{+\frac{2}{3}}\left[_{+4} \oplus\right](\overline{3}, 2)_{+\frac{5}{6}}\left[_{+4} \oplus\right](1,1)_{+1}\left[_{+4}+\right. \\
](\overline{3}, 1)_{-\frac{2}{3}}\left[{ }_{-4} \oplus\right](3,2)_{-\frac{5}{6}}\left[_{-4} \oplus\right](1,1)_{-1}\left[{ }_{-4}+\right. \\
\left.\left.\oplus](8,1)_{0}\right]_{0} \oplus\right](\overline{3}, 2)_{-\frac{1}{6}}\left[_{0} \oplus\right](3,2)_{+\frac{1}{6}}\left[_{0} \oplus\right. \\
\oplus](1,3)_{0}\left[_{0} \oplus\right](1,1)_{0}\left[_{0} \oplus\right](1,1)_{0}[0
\end{array}\right]
$$


Alternatively we can describe the decomposition of $\mathrm{Adj}(\mathrm{SO} 10)$ as displayed in eq. 58 by the irreducible representations of SU5 and the eigenvalues of $\mathbf{J}$

$$
\operatorname{Adj}\left(\text { SO10) }=\left[\begin{array}{c}
\{\overline{10}\}_{4} \oplus\{10\}_{-4} \oplus \\
\oplus\{24\}_{0} \oplus\{1\}_{0}
\end{array}\right] ;\{24\}_{0}=\operatorname{Adj}(\text { SU5) }\right.
$$

with the identification through eq. 58

$$
\begin{aligned}
& \left.\{\overline{10}\}_{+4}=\right](\overline{3}, 1)_{-\frac{2}{3}}\left[{ }_{-4} \oplus\right](3,2)_{-\frac{5}{6}}\left[_{-4} \oplus\right](1,1)_{-1}\left[{ }_{-4}\right. \\
& \left.\{10\}_{-4}=\right](3,1)_{+\frac{2}{3}}\left[_{+4} \oplus\right](\overline{3}, 2)_{+\frac{5}{6}}\left[_{+4} \oplus\right](1,1)_{+1}\left[{ }_{+4}\right.
\end{aligned}
$$

and

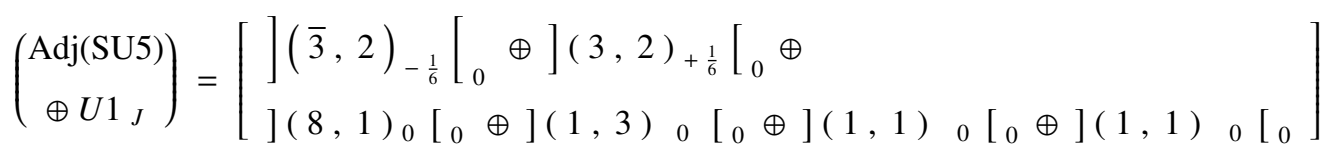

We wish to complement the structure of $\operatorname{Adj}(S U 5) \oplus U 1_{J}$ in eq. 61 with the numbers of gauge bosons first without letting them acquire mass:

$$
\left(\begin{array}{c}
\operatorname{Adj}(\text { SU5) } \\
\oplus U 1_{J}
\end{array}\right)=\left[\begin{array}{rrr}
\#_{t o t} & \#_{\text {tot }}^{B S M} \\
\hline \overline{6} \oplus 6 & 12 & 12 \\
8\left|S U 3_{c} \oplus 3\right| S U 2_{L} \oplus 2 \mid U 1_{y \oplus U 1_{J}} & 13 & 1
\end{array}\right]
$$

Together with the 20 BSM gauge bosons displayed in eq. 60 the total count of these modes, all necessarily obtaining their mass from the primary breakdown as described in section 2-1 yield 33 gauge bosons $\rightarrow 99$ degrees of freedom. Some of them induce baryon- and lepton-number violation and thus are required to be much heavier than the elcroweak scale - reaching up to approximatively $10^{16} \mathrm{GeV}$.

With this partial characterization of the so induced minimal gauge boson collection beyond the standard model, we end the corresponding point 2 , for the sake of clarity and conciseness of presentation this 'Synthesis' section, thereby omitting the (anti-)neutrino mass generation, the first definite detection of BSM physics.

\section{Acknowledgement}

It is a pleasure to thank Larissa Brawina, Yiota Foka and Sonia Kabana for orchestring this conference in a constructive and lively atmosphere.

\section{References}

[1-2013] François Englert, Nobel lecture, 'The BEH Mechanism and its Scalar Boson', http://www.nobelprize.org/nobel_prizes/physics/laureates/2013/englert-lecture.html in file: englert-lecture-slides.pdf .

[2-2013] Peter Higgs, Nobel lecture, 'Evading the Goldstone Theorem', http://www.nobelprize.org/nobel_prizes/physics/laureates/2013/higgs-lecture.html in file: higgs-lecture.pdf . 
[3-1975] H. Fritzsch and P. Minkowski, 'Unified interactions of leptons and hadrons', Annals Phys.93 (1975) 193 and H. Georgi, 'The state of the art - gauge theories', AIP Conf.Proc.23 (1975) 575.

[4-2008] P. Minkowski, 'The origin of neutrino mass - stations along the path of cognition', Contribution to - Discrete'08 - Symposium on the Prospects in the Physics of Discrete Symmetries 11.-16. December 2008, IFIC, Valencia, Spain, J.Phys.Conf.Ser. 171 (2009) 012016, http://www.mink.itp.unibe.ch/lectures.html , in file: nuorigin08.pdf .

[5-1976] F. Gursey, Pierre Ramond and P. Sikivie, 'A universal gauge theory model based on E6', YALE-3075-118, Sep 1975, Phys.Lett.B60 (1976) 177.

[6-1980] F. Gursey, 'Exceptional Groups for Guts and Remarks on Octonions', published in *Baltimore 1980, Proceedings, Weak Interactions As Probes Of Unification*, 635-646.

[7-1984] Luis Alvarez-Gaume (Harvard U.) and Edward Witten (Princeton U.). Aug 1983., 111 pp., 'Gravitational Anomalies', Nucl.Phys. B234 (1984) 269, HUTP-83/A039, DOI: 10.1016/05503213(84)90066-X.

[8-2012] Michael B. Green, John H. Schwarz, Edward Witten, 'Superstring Theory, 2 Volume Set 25th Anniversary Edition, Cambridge Monographs on Mathematical Physics 2012.

[9-1995] M. E. Peskin, D. V. Schroeder, 'An Introduction to quantum field theory', Addison-Wesley, Reading, MA 1995x .

[10-1013] K.S. Babu, E. Kearns, U. Al-Binni, S. Banerjee, D.V. Baxter, Z. Berezhiani, M. Bergevin, S. Bhattacharya, S. Brice, R. Brock et al., 'Baryon Number Violation', Nov 20, 2013. 39 pp., arXiv:1311.5285 [hep-ph].

[11-2014] Guido Altarelli, 'The Higgs and the Excessive Success of the Standard Model', Jul 8, 2014. 19 pp., RM3-TH-14-12, CERN-PH-TH-2014-127, Conference: C14-05-18.1, arXiv:1407.2122 [hep-ph].

[12-2014] Ferruccio Feruglio, Ketan M. Patel, Denise Vicino, 'Order and Anarchy hand in hand in 5D SO(10)', Jul 10, 2014. 29 pp., arXiv:1407.2913 [hep-ph].

[13-2013] 'Tests of conservation laws', updated October 2013 by L. Wolfenstein (Carnegie-Mellon University) and C.-J. Lin (LBNL), PDG review, J. Beringer et al. (PDG), PR D86, 010001 (2012) and 2013 update for the 2014 edition (URL: http://pdg.lbl.gov).

[14-1927] F. Peter und H. Weyl, 'Die Vollständigkeit der primitiven Darstellungen einer geschlossenen kontinuierlichen Gruppe', Ann. Math. 97 (1927) 737-755.

[15-2013] J. Ellis, 'Higgs Physics', Dec 19, 2013, 52 pp., KCL-PH-TH-2013-49, LCTS-2013-36, CERN-PH-TH-2013-315, Conference: C13-06-05, arXiv:1312.5672 [hep-ph].

[16-1974] J.J. Aubert (MIT, LNS) et al., E598 Collaboration, 'Experimental Observation of a Heavy Particle J', Print-74-1663 (MIT) 1974. 9 pp., Phys.Rev.Lett. 33 (1974) 1404-1406, COO-3069271, DOI: 10.1103/PhysRevLett.33.1404.

[17-1974] J.E. Augustin (SLAC) et al., SLAC-SP-017 Collaboration, 'Discovery of a Narrow Resonance in e+ e- Annihilation', SLAC-PUB-1504, Nov 1974, 6 pp., Phys.Rev.Lett. 33 (1974) 1406-1408, DOI: 10.1103/PhysRevLett.33.1406 . 